\title{
REFLEXIONES SOBRE EL RELEVAMIENTO Y ANÁLISIS COMPARATIVO DE PATOLOGÍAS OSTEOARTICULARES EN RESTOS ESQUELETALES HUMANOS
}

\author{
Leandro Luna ${ }^{1 *}$, Claudia Aranda² y Alexandrina Amorim Alves ${ }^{3}$

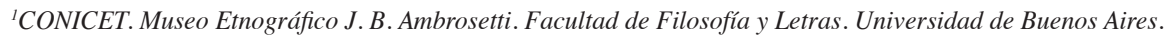 \\ ${ }^{2}$ Museo Etnográfico J. B. Ambrosetti. Facultad de Filosofía y Letras. Universidad de Buenos Aires. \\ ${ }^{3}$ Departamento CIAS. Centro de Investigación en Antropología y Salud. Universidad de Coimbra. Portugal
}

PALABRAS CLAVE paleopatología; bioarqueología; registro consensuado; lesiones osteoarticulares; esqueletos

RESUMEN Desde los inicios de la bioarqueología, los estudios de las alteraciones osteoarticulares utilizan definiciones, protocolos de relevamiento y propuestas de análisis de los datos muy variados, lo cual generalmente imposibilita su adecuada comparación. En este trabajo se discuten algunos problemas derivados de esa diversidad de perspectivas, con el objetivo de plantear la necesidad de formular criterios consensuados de registro y análisis que permitan generar datos comparables. Se reflexiona sobre aspectos relativos al proceso de relevamiento de los indicadores osteoarticulares y de procesamiento de la información, al mismo tiempo que se plantean opciones terminológicas y procedimentales que contribuyan a generar un marco general para el desarrollo de estudios multimuestrales. Rev Arg Antrop Biol 19(1), 2017. doi:10.17139/ raab.2017.0019.01.08

\section{KEY WORDS paleopathology; bioarchaeology; agreed recording; osteoarticular lesions; skeletons}

ABSTRACT Since the beginnings of bioarchaeology, studies of osteoarticular disorders make use of diverse definitions, survey protocols, and analysis proposals; this heterogeneity usually prevents adequate comparisons among them. This paper discusses a number of problems arising from the diversity of perspectives adopted, with the aim of highlighting the need to develop consensus for recording and analyzing data in order to generate comparable criteria. It reflects on certain aspects related to the process of recording musculoskeletal indicators, as well as to information processing. At the same time it offers terminological and procedural options intended to contribute to the generation of a general framework for the development of multi-sample studies. Rev Arg Antrop Biol 19(1), 2017. doi:10.17139/raab.2017.0019.01.08
Los estudios bioarqueológicos que focalizan en el análisis de las alteraciones osteoarticulares, entendidas como cualquier modificación tisular que afecta la estructura cortical de las articulaciones, abordan una gran variedad de problemáticas, con líneas de interpretación y protocolos de relevamiento muy diversos. Dicha multiplicidad socava las posibilidades de realizar análisis intermuestrales de amplia escala ya que los datos básicos generados no son, en la mayoría de los casos, compatibles desde un punto de vista comparativo. Uno de los principales dilemas que enfrenta la bioarqueología actual tiene que ver con la falta de acuerdo en la generación y aplicación de estrategias metodológicas por parte de diferentes grupos de investigación (Luna et al., 2014). Deben destacarse, como antecedentes en este sentido, algunas contribuciones que pretendieron estandarizar criterios de relevamiento e interpretación en las diferentes temáticas dentro de la bioarqueología y la paleopatología (e.g. Buikstra y Ubelaker, 1994; Brickley y Mckinley, 2004; Steckel et al., 2005), mientras que otras avanzaron en ámbitos de interés específicos como la paleodemografía (Ferembach et al., 1980; Hoppa y Vaupel, 2002) y los cambios entésicos (Villotte y Knüsel, 2013; Villotte et al., 2016) Este trabajo nace de la necesidad de reflexionar acerca de la importancia de formular criterios adecuados para el registro y análisis de las alteraciones osteoarticulares en restos esqueletales humanos ${ }^{1}$, especialmente aquellas derivadas de procesos crónicos de estrés mecánico, de manera que los procedimientos implementados tengan una estructura uniforme que permita arribar a resultados comparables a partir de muestras de diferentes procedencias y

*Correspondencia a: Leandro Luna. Museo Etnográfico J. B. Ambrosetti, Facultad de Filosofía y Letras. Universidad de Buenos Aires. Moreno 350 (1091). Ciudad Autónoma de Buenos Aires. E-mail: lunaranda@gmail.com

Financiamiento: sin financiamiento.

Recibido 9 Enero 2016; aceptado 22 Marzo 2016

doi:10.17139/raab.2017.0019.01.08 
de estudios realizados por diferentes investigadores. De esta forma, el desarrollo de propuestas de consenso para el relevamiento de restos bioantropológicos, tanto arqueológicos como contemporáneos, sigue siendo un desafío pendiente que debe ser abordado en el corto plazo en el ámbito de la disciplina.

La diversidad de metodologías disponibles para el registro de los indicadores osteoarticulares de estrés mecánico incluye protocolos que generalmente muestran incompatibilidades, ambigüedades y contradicciones internas, las cuales deben ser superadas para asegurar aproximaciones comparativas efectivas y de amplia escala. En este sentido, el objetivo de este trabajo es identificar los principales problemas que presentan los estudios bioarqueológicos de patologías articulares desde una perspectiva metodológica, para contribuir a la generación de procedimientos unificados de registro, análisis e interpretación (Buikstra y Ubelaker, 1994; Brickley y McKinley, 2004). Para ello, se plantea la discusión de dos ejes principales, referidos al proceso de relevamiento de los indicadores osteoarticulares (definiciones de las variables, categorizaciones aplicadas y los problemas de la diversidad de las manifestaciones) y al procesamiento de la información (intraesqueletal, intramuestral o intermuestral; prevalencias de las lesiones; conjuntos articulares comparados). Se trata de una primera aproximación que no pretende arribar a conclusiones definitivas sino a abrir el panorama de posibilidades que permita expandir la variedad de enfoques y orientar hacia una homogeneización de los procedimientos.

\section{Terminología y definiciones}

En primer lugar, resulta importante reflexionar sobre la importancia de enunciar definiciones claras y precisas de lo que se pretende estudiar. Se han realizado numerosas contribuciones que buscaron describir y definir tanto las características macroscópicas como la etiología multifactorial de las alteraciones osteoarticulares desde perspectivas clínicas, bioquímicas, genéticas y forenses (e.g. Masoro y Austad, 2006; Seibel et al., 2006; Pinhasi y Mays, 2008; Robson Brown et al., 2008). La anormalidad degenerativa más común suele ser la formación de osteofitos a lo largo de los márgenes articulares, resultado del estrés funcional por tracciones capsulares sistemáticas, principalmente en huesos de las manos, rodilla y cadera para individuos mayores a los 50 años de edad; los casos de eburnación, que se identifican tanto escópica como radiográficamente en la forma de áreas de esclerosis subcondral y la presencia de porosidades intraarticulares, que suelen ser menos frecuentes en el ser humano, aunque sus prevalencias pueden superar el $40 \%$ en adultos maduros (Lawrence et al., 1966; Kerr et al., 1985; Petersson, 1996; Hough, 2001; Solomon, 2001; Lagier, 2006).

En la literatura bioarqueológica, las patologías articulares son definidas de muy diferente manera, con términos que generalmente no son sinónimos lo cual genera confusión heurística (Ortner, 2003). Además, algunas definiciones no son operacionalmente adecuadas, ya que no presentan una relación lógica con lo que efectivamente puede ser observado y analizado desde el registro bioarqueológico. Se suelen utilizar términos como artritis, osteoartritis, artrosis, osteoartrosis, enfermedad degenerativa articular, artropatía degenerativa, artropatía proliferativa, patología articular degenerativa o artritis degenerativa, entre otros (Jurmain, 1977; Bridges, 1992; Knusel, 1993; Buikstra y Ubelaker, 1994; Rogers y Waldron, 1995; Mays, 2002; Ortner, 2003; Steckel et al., 2005; Roberts y Manchester, 2005; Helmtrud y Tilley, 2007; Lieverse et al., 2007; Weiss y Jurmain, 2007; Luna, 2008; Rojas Sepúlveda et al., 2008; Waldron, 2009, 2012; Scabuzzo, 2012), siendo el de osteoartritis el más utilizado, sobre todo por la vertiente norteamericana de la disciplina (Weiss y Jurmain, 2007) y etimológicamente indica una inflamación articular del hueso (Neumann, 1999; Manuila et al., 2000). Esta definición no guarda relación directa con los diferentes tipos de reacciones óseas pasibles de ser observadas en el registro osteológico. El término es indicativo de una patología inflamatoria de la articulación, la cual si bien puede producir consecuencias importantes en el estilo de vida de los individuos que la padecen, no necesariamente deja improntas apreciables en el tejido óseo. Además, gran cantidad de estudios clínicos documentan una alta diversidad etiológica, generalmente de índole infecciosa o congénita (Kellgren et al., 1963; Ortner, 2003; Rogers et al., 2004; Roberts y Manchester, 2005; Plischuk, 
2012), lo cual hace muy dificultosa su identificación precisa en los conjuntos esqueletales. En este sentido, la inespecificidad de muchas de las patologías articulares generalmente imposibilita un diagnóstico diferencial ajustado.

Por otro lado, el término osteoartrosis o enfermedad degenerativa articular, pone énfasis en el componente degenerativo de la articulación, se focaliza en el carácter proliferativo de la patología y en general denota una ausencia de respuesta inflamatoria como causa primaria principal (Neumann, 1999; Manuila et al., 2000). Este término resulta más adecuado para describir e interpretar las manifestaciones patológicas articulares que sistemáticamente se observan a nivel esqueletal. La osteoartrosis es entonces entendida como el derivado de una enfermedad crónica degenerativa, no necesariamente ligada a un origen inflamatorio, que produce la alteración destructiva de los cartílagos articulares y la formación de hueso nuevo en la región subcondral, específicamente en los márgenes articulares, como consecuencia de situaciones crónicas de estrés mecánico (Foss y Byers, 1972; Jurmain, 1990; Aufderheide y Rodríguez-Martín, 1998; Ortner, 2003; Lieverse et al., 2007). Las proliferaciones óseas suelen recibir el nombre de labiaciones, osteofitos o lipping, y también pueden estar acompañadas de esclerosis (endurecimiento del tejido óseo), eburnación (aspecto brilloso derivado del contacto directo de las superficies articulares por eliminación total del cartílago) o anquilosis (unión permanente de los huesos de una articulación) (Rogers et al., 1987; Aufderheide y Rodríguez-Martín, 1998; Miles, 1999; Mays, 2002; Waldron, 2009; Roberts y Manchester, 2005; Lagier, 2006). Cabe destacar que esta descripción no puntualiza en las causas específicas de la manifestación, sino en las características escópicas de las lesiones. De esta manera, a pesar de su uso extendido en bioarqueología, se desaconseja la utilización del término osteoartritis, ya que puede llevar a errores de interpretación y definición de los procesos analizados y se propone reemplazarlo por el de osteoartrosis o enfermedad degenerativa articular, que se restringe a la definición de alteraciones proliferativas periarticulares de carácter mecánico crónico, directamente asociados al uso del cuerpo y a la edad del individuo (Miles, 1999; Resnick y Kransdorf, 2005; Weiss yJurmain, 2007; Berenbaum, 2013).

Otra manifestación patológica que suele identificarse en el interior de la superficie articular es la porosidad, producida en este caso sí por un proceso inflamatorio óseo y cartilaginoso. Generalmente se observa un deterioro cortical con erosión, derivado de una inflamación inicial de los tejidos duros con posterior afectación cartilaginosa (Rothschild, 1997). Esta porosidad puede aparecer en forma incipiente en un sector acotado de la articulación, o en forma masiva ocupando toda la zona. Es importante considerar que algunos autores (e.g. Rothschild, 1997; Sofaer Derevenski, 2000) consideran que esta variable no está relacionada con la enfermedad degenerativa articular, y que en consecuencia debe ser excluida de los análisis ya que la identificación aislada de porosidad no es suficiente para inferir la patología. Asimismo, puede ser confundida con deterioros tafonómicos y de esa manera introducir factores de variación ajenos al problema abordado (Rojas Sepúlveda et al., 2008). En consecuencia, se propone que la porosidad y las proliferaciones degenerativas deben ser analizadas e interpretadas por separado, ya que dan cuenta de procesos de deterioro articular diferentes, que pueden o no actuar en forma sincrónica (Rothschild, 1997). La identificación de las dos condiciones en una misma articulación suele ser común (Fig. 1), indicando la acción simultánea de alteraciones inflamatorias y de estrés mecánico.

\section{Hacia un consenso metodológico}

Merecen discutirse las características del protocolo metodológico aplicado, de manera de llegar a consensos generales sobre el relevamiento de las variables relacionadas con la enfermedad osteoarticular. En la literatura especializada existe una gran diversidad de propuestas de relevamiento y categorizaciones, así como también ambigüedades en las definiciones acerca de qué se debe observar en cada caso (e.g. Säger, 1969; Jurmain, 1977, 1990; Waldrony Rogers, 1991;Bridges, 1994;Buikstray Ubelaker, 1994; Lovell, 1994; Dawson y Trinkaus, 1997; Knusel et al., 1997; Brickley y Waldron, 1998; Miles, 1999; Sofaer Derevenski, 2000; Crubézy et al., 2002; Cope et al., 2005; Rojas-Sepúlveda et al., 2008; Waldron, 2009; Rando y Waldron, 2012). Resulta fundamental explicitar con claridad y detenimiento cómo se 


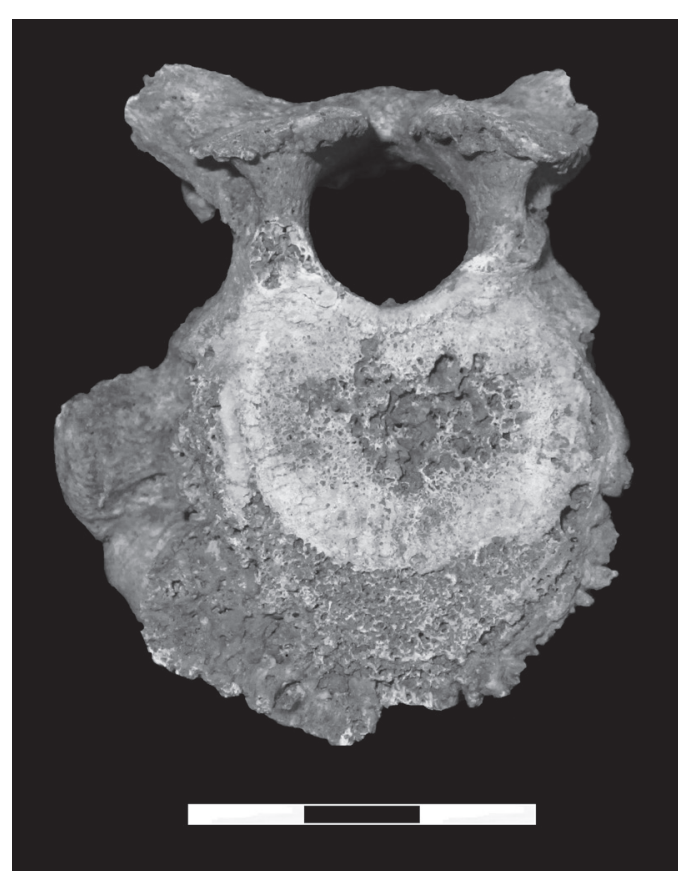

Fig. 1. Osteoartrosis y porosidad identificada en una misma articulación (cara superior de cuerpo vertebral dorsal).

definen las categorías operativas en las publicaciones científicas (Jurmain y Kilgore, 1995), de manera de robustecer las interpretaciones y permitir la realización de estudios comparativos. Sin embargo, usualmente los trabajos dedican poco espacio a estos temas, con definiciones superficiales y poco claras, lo cual hace dificultoso comprender cómo se discriminó entre cada categoría considerada y genera tanto un alto componente de subjetividad impuesto por el investigador como una baja solidez de los resultados [e.g., Bridges (1991) considera, 0: sin artritis; 1: mínima; 2: leve; 3: moderada; 4: severa]. Entre la gran cantidad de propuestas, por ejemplo Jurmain (1990) define con detalle cada una de las cuatro categorías elegidas para relevar la osteoartrosis, aunque no considera el porcentaje de la superficie articular afectada. Por otro lado, Rodrígues Carvalho (2004) incluye la proliferación y la porosidad en el mismo esquema clasificatorio, y cuantifica la intensidad de las lesiones periarticulares a través de la medición de las longitudes máximas observadas. Este último punto es especialmente importante para mejorar el poder descriptivo de las variables relevadas.
Un avance en este problema es la publicación de imágenes que complementen las descripciones de cada categoría. Por ejemplo, Rojas Sepúlveda et al. (2008) incorporan este recurso para describir con precisión tres variables diferentes para los cuerpos vertebrales (osteofitos, lipping y porosidad) y para las articulaciones del arco dorsal (lipping, pitting y eburnación), las cuales analizan tanto en conjunto como separadamente.

En resumen, para el desarrollo de un protocolo consensuado se vuelve imprescindible definir y discriminar claramente cada una de las categorías, evitar superposiciones y ambigüedades en su definición, e intentar establecer criterios cuantitativos de discriminación (Jurmain y Kilgore, 1995). Para el relevamiento de las proliferaciones periarticulares se propone la siguiente categorización: 0) ninguna indicación de osteoartrosis; 1) se evidencian irregularidades incipientes en la zona periarticular, menores a $1 \mathrm{~mm}$ de longitud; 2) los osteofitos son claramente visualizables y no exceden los cinco $\mathrm{mm}$ de longitud; 3) las excrecencias tienen una longitud que excede los cinco $\mathrm{mm}$, lo que se corresponde con una afección grave de la articulación (Fig. 2) (Luna, 2008). La presencia de eburnación y anquilosis pueden ser también incluidas en la última categoría. Por otra parte, para la porosidad se propone considerar la proporción del tejido intraarticular afectado de la siguiente manera: 0) sin porosidad; 1) menos de un tercio de la superficie afectada; 2) entre el un tercio y dos tercios; 3) más de dos tercios (Fig. 3). Se considera que estas categorizaciones son lo suficientemente amplias como para minimizar el error generado por el investigador, al mismo tiempo que permiten obtener información valiosa para realizar interpretaciones adecuadas.

\section{Variaciones intraarticulares}

Otro aspecto a discutir se refiere a cuáles son los procedimientos más adecuados para el relevamiento de articulaciones que presentan manifestaciones osteoartrósicas de intensidad variable, como suele identificarse en los cuerpos vertebrales. En general, los investigadores optan por asignar el grado más alto registrado, perdiéndose de esa manera información sobre la variabilidad existente. Como aproximación 


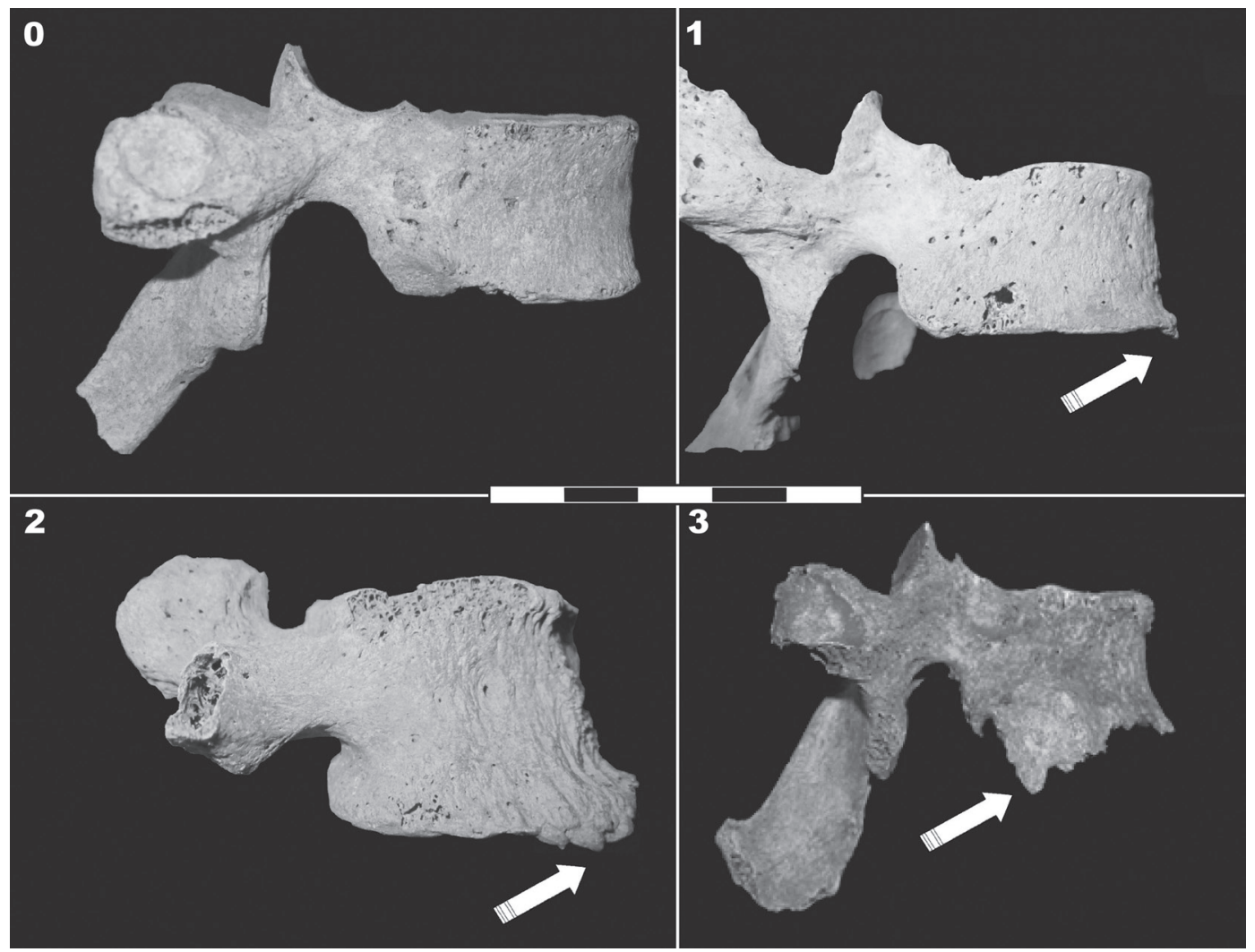

Fig. 2. Categorización de la osteoartrosis en cuerpos vertebrales según la intensidad de la proliferación ósea.

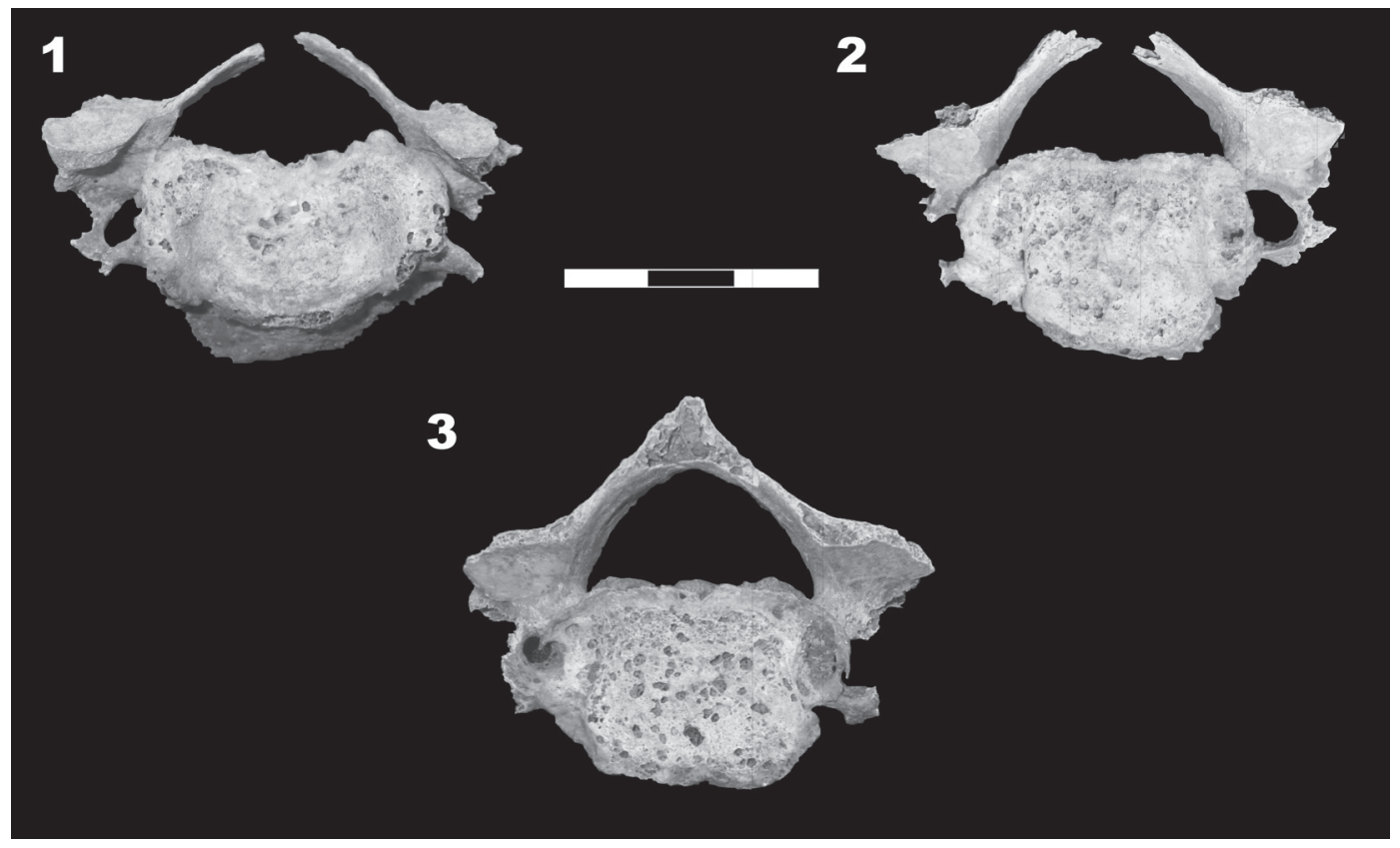

Fig. 3. Categorización de la porosidad según el porcentaje de la superficie articular involucrada (cara superior de vértebras cervicales). 
alternativa para arribar a resultados más adecuados, se propone relevar tanto la intensidad como el porcentaje de afectación dividiendo la articulación en cuatro cuadrantes ortogonales (e.g. Hayeri et al., 2010). Para caracterizar la intensidad, se propone asignar en primer lugar un valor entre 0 y 3 a cada cuadrante, considerando las categorizaciones antes mencionadas y luego sumar las cifras obtenidas para obtener valores de intensidad entre 0 y 12 para cada articulación. A su vez, con el objetivo de considerar los casos en los que las zonas articulares se encuentran deterioradas o ausentes por los efectos de agentes postdepositacionales, debe calcularse la razón entre ese valor final y la cantidad de cuadrantes efectivamente relevados. Con respecto a los porcentajes de afectación de cada cuadrante, el procedimiento es similar, considerando cuatro categorías ( 0 : no afectado; 1 : menos de un tercio del borde periarticular afectado; 2: entre un tercio y dos tercios; 3 : más de dos tercios).

Siguiendo el mismo razonamiento, es posible calcular valores para las diferentes porciones anatómicas de un mismo individuo, considerando tanto la intensidad y el porcentaje de afectación como la cantidad de elementos disponibles. En este caso, los valores parciales obtenidos para los elementos considerados deben ser sumados y divididos por la cantidad de superficies articulares relevadas. En un nivel de agregación mayor de los datos, se pueden realizar análisis comparativos con diferentes conjuntos muestrales, por ejemplo entre individuos masculinos y femeninos incluidos dentro de un determinado rango de edad, o del mismo sexo y rango de edad pero de diferentes procedencias. A pesar de que este procedimiento implica una cantidad mayor de tiempo invertido en el relevamiento, se considera que los resultados obtenidos son más precisos y permiten conocer los patrones de proliferación ósea con mayor detalle, mejorando las posibilidades de realizar comparaciones intra e intermuestrales.

\section{Análisis de los datos}

Por último, es necesario discutir cómo se analiza la información obtenida. Las comparaciones que no consideran la variación generada por las categorías de sexo y edad (e.g. adultos jóvenes -entre 20 y 34 años-, adultos medios -en- tre 35 y 49 años- y adultos maduros -mayores de 49 años; Buikstra y Ubelaker, 1994; ver Hoppa y Vaupel, 2002) ocultan la diversidad interna existente y solo ofrecen información superficial y sesgada para caracterizar los modos de vida de las sociedades del pasado (Jurmain, 1977; Rojas Sepúlveda et al., 2008). Dado que esas dos variables demográficas tienen un impacto muy importante en los patrones observados, solo resultan interesantes las interpretaciones que evalúan en forma comparativa las mismas categorías de edad entre muestras de diferentes sexos o procedencias. A su vez, debería analizarse cuáles son, dentro de cada submuestra, las articulaciones más afectadas (en superficie y en intensidad) o qué submuestra es afectada en una mayor cantidad de articulaciones. En este sentido, se considera fundamental precisar las variaciones discriminando las diferentes partes anatómicas y realizar comparaciones según la cantidad de articulaciones afectadas y la intensidad de las lesiones identificadas. Es en la concatenación de todas estas perspectivas donde se pueden identificar tendencias interesantes sobre los estilos de vida de las poblaciones del pasado. Por último, resulta también muy importante explicitar en cada presentación cuáles son los niveles de análisis considerados (por articulación, por grupo articular, por individuo, por grupos de individuos, por sexo, por edad, por cronología u otras), anclar las comparaciones por grupos de edad y asegurar frecuencias relativamente similares para cada submuestra.

\section{Palabras finales}

Este trabajo solo pretende enunciar los aspectos más importantes que se sugiere deben ser considerados como interrogantes sobre las diferentes etapas del proceso de análisis; el desarrollo de una discusión exhaustiva y profunda de cada uno de los problemas que surgen en torno a los estudios sobre las alteraciones osteoarticulares va mucho más allá de su contenido. El objetivo principal es promover la reflexión entre los especialistas en la temática, de manera de avanzar hacia un consenso general que permita llevar adelante estudios comparativos, ya que el poder explicativo de las interpretaciones efectuadas se ve fuertemente influenciado por la elaboración previa de un adecuado diseño 
de relevamiento. Es por este motivo que resulta fundamental crear protocolos de registro compatibles entre los investigadores para garantizar la comparabilidad entre muestras y la calidad de los resultados obtenidos.

\section{AGRADECIMIENTOS}

Agradecemos los comentarios de los dos evaluadores de este trabajo.

\section{Nota}

${ }^{1}$ Una versión previa de este trabajo ha sido presentada en el "II Taller Nacional de Bioarqueología y Paleopatología" celebrado en Rosario (Santa Fé), los días 29 y 30 de mayo de 2014.

\section{LITERATURA CITADA}

Aufderheide A, Rodriguez-Martín G. 1998. The Cambridge encyclopedia of human pathology. Cambridge: Cambridge University Press.

Berenbaum F. 2013. Osteoarthritis as an inflammatory disease (osteoarthritis is not osteoarthrosis!). Osteoarthritis Cartilage 21(1):16-21. doi: 10.1016/j.joca.2012.11.012

Brickley M, McKinley J. 2004. Guidelines to the standard of recording human remains. IFA papers $\mathrm{N}^{\circ} 7$. Southampton: British Association for Biological Anthropology and Osteoarchaeology.

Brickley M, Waldron T. 1998. Relationship between bone density and osteoarthritis in a skeletal population from London. Bone 22(3):279-283. doi:10.1016/S87563282(97)00272-X

Bridges P. 1991. Degenerative joint disease in hunter-gatherers and agriculturalists from the southeastern United States. Am J Phys Anthropol 85:379-391. doi:10.1002/ ajpa. 1330850403

Bridges P. 1992. Prehistoric arthritis in the Americas. Ann Rev Anthropol 21:67-91. doi:10.1146/annurev. an.21.100192.000435

Bridges, P. 1994. Vertebral arthritis and physical activities in the prehistoric southeastern United States. Am J Phys Anthropol 93:83-93. doi:10.1002/ajpa.1330930106

Buikstra J, Ubelaker, D. 1994. Standards for data collection from human skeletal remains. Arkansas Archaeological Survey Research Series No. 44. Faytteville, Arkansas.

Cope J, Berryman A, Martin D, Potts D. 2005. Robusticity and osteoarthritis at the trapeziometacarpal joint in a Bronze Age population from Tell Abraq, United Arab Emirates. Am J Phys Anthropol 126:391-400. doi:10.1002/ajpa.20097

Crubézy E, Goulet J, Bruzek J, Jelinek J, Rougé D, Ludes, B. 2002. Epidemiology of osteoarthritis and enthesopathies in a Europe and population dating back 7700 years. Joint Bone Spine 69:580-588. doi:10.1016/ S1297-319X(02)00455-4

Dawson J, Trinkaus E. 1997. Vertebral osteoarthritis of the La Chapelle-auz-Saints 1 Neanderthal. J Arch Sci 24:1015-1021. doi:10.1006/jasc. 1996.0179

Ferembach D, Schwidetzky I, Stloukal M. 1980. Recommendations for age and sex diagnoses of skeletons. J Hum
Evol 9:517-549. doi:10.1016/0047-2484(80)90061-5

Foss M, Byers P. 1972. Bone density, osteoarthrosis of the hip, and fracture of the upper end of the femur. Ann Rheum Dis 31:259-264. doi:10.1136/ard.31.4.259

Hayeri M, Shiehmorteza M, Trudell D, Hefflin T, Resnick D. 2010. Proximal tibial osteophytes and their relationship with the height of the tibial spines of the intercondylar eminence: paleopathological study. Skeletal Radiol 39:877-881. doi:10.1007/s00256-009-0838-z

Helmtrud I, Tilley S. 2007. The pathogenesis of oseoarthritis. En: Bronner F, Farach-Carson M, editores. Bone and osteoarthritis. Topics in bone biology. Connecticut: University of Connecticut. Volume 4. p 1-18.

Hoppa R, Vaupel J.. 2002. Paleodemography. Age distributions from skeletal samples. Cambridge: Cambridge University Press.

Hough A. 2001. Pathology of osteoarthritis. En: Koopman W, editor. Arthritis and allied conditions. Philadelphia: Lippincott Williams and Wilkins. p. 2167-2194.

Jurmain R. 1977. Stress and the etiology of osteoarthritis. Am J Phys Anthropol 46:353-366. doi:10.1002/ ajpa.1330460214

Jurmain R. 1990. Paleoepidemiology of a central California prehistoric population from CA-ALA-329: II. Degenerative disease. Am J Phys Anthropol 83:83-94. doi:10.1002/ajpa.1330830110

Jurmain R, Kilgore L. 1995. Skeletal evidence of osteoarthritis: a paleopathological perspective. Ann Rheum Dis 54:443-450. doi:10.1136/ard.54.6.443

Kellgren J, Lawrence J, Bier F. 1963. Genetic factors in generalized osteo-arthrosis. Ann Rheum Dis 22:237-255. doi:10.1136/ard.22.4.237

Kerr R, Resnick D, Pineda C, Haghighi P. 1985. Osteoarthritis of the glenohumeral joint: a radiologic-pathologic study. Am J Roentgen 144:967-972. doi:10.2214/ ajr.144.5.967

Knusel C. 1993. On the biomechanical and osteoarthritic differences between hunter-gatherers and agriculturalists. Am J Phys Anthropol 91:523-527. doi:10.1002/ ajpa.1330910409

Knusel C, Goggel S, Lucy D. 1997. Comparative degenerative joint disease of the vertebral column in the medieval monastic cemetery of the Gilbertine Priory of St. Andrew, Fishergate, York, England. Am J Phys Anthropol 103:481-195. doi:10.1002/(SICI)10968644(199708)103:4<481::AID-AJPA6>3.0.CO;2-Q

Lagier R. 2006. Bone eburnation in rheumatic diseases: a guiding trace in today's radiological diagnosis and in paleopathology. Clin Rheumatol 25:127-131. doi:10.1007/s10067-004-1055-8

Lawrence J, Bremner J, Bier F. 1966. Osteo-arthrosis prevalence in the population and relationship between symptoms and X-Ray changes. Ann Rheum Dis 25:1-24. doi:10.1136/ard.25.1.1

Lieverse A, Weber A, Bazaliiskiy V, Goriunova O, Savel'ev N. 2007. Osteoarthritis in Siberia's Cis-Baikal: skeletal indicators of hunter-gatherer adaptation and cultural change. Am J Phys Anthropol 132:1-16. doi:10.1002/ ajpa. 20479

Lovell N. 1994. Spinal arthritis and physical stress at Bronze Age Harappa. Am J Phys Anthropol 93:149-164. doi:10.1002/ajpa.1330930202

Luna L. 2008. Estructura demográfica, estilo de vida y relaciones biológicas de cazadores-recolectores en un ambiente de desierto. Sitio Chenque I (Parque Nacional Lihué Calel, provincia de La Pampa). BAR International Series 1886. Oxford: Archaeopress.

Luna L, Aranda C, Suby J. 2014. Hacia la generación de un 
protocolo consensuado de relevamiento en bioarqueología. Resultados, avances y discusiones del Primer Taller Nacional de Bioarqueología y Paleopatología. Intersecciones Antropol 15:485-489.

Manuila L, Manuila A, Lewalle P, Nicoulin M. 2000. Dicionário médico. Lisboa: Climepsi Editores.

Mays S. 2002. The archaeology of human bones. Nueva York: Routledge.

Masoro E, Austad, S. 2006. Handbook of the biology of aging. San Diego: Academic Press.

Miles A. 1999. A five-grade categorization of age-related change in the acromio-clavicular joint derived from the skeletal remains of early 19th Century Londoners of known sex and age. Int J Osteoarchaeol 9:83-101. doi:10.1002/(SICI)10991212(199903/04)9:2<83::AID-OA461>3.0.CO;2-V

Neumann D. 1999. Joint deformity and dysfunction: a basic review of underlying mechanism. Arthritis Care \& Research 12(2):139-151. doi:10.1002/15290131(199904)12:2<139::AID-ART9>3.0.CO;2-7

Ortner D. 2003. Identification of pathological conditions in human skeletal remains. Florida: Academic Press.

Petersson I. 1996. Occurrence of osteoarthritis of the peripheral joints in European populations. Ann Rheum Dis 55:659-664. doi:10.1136/ard.55.9.659

Pinhasi R, Mays S. 2008. Advances in human palaeopathology. Chichester: John Wiley \& Sons Ltd.

Plischuk M. 2012. Detección y diagnóstico de patologías en restos óseos humanos: aproximación epidemiológica a una muestra documentada. Tesis Doctoral inédita. Facultad de Ciencias Naturales y Museo. Universidad Nacional de La Plata.

Rando C, Waldron T. 2012. TMJ osteoarthritis: a new approach to diagnosis. Am J Phys Anthropol 148:45-53. doi:10.1002/ajpa.22039

Resnick D, Kransdorf M. 2005. Bone and joint imaging. Nueva York: Elsevier.

Roberts C, Manchester K. 2005. The archaeology of disease. Nueva York: Cornell University Press.

Robson Brown K, Pollintine P, Adams M. 2008. Biomechanical implications of degenerative joint disease in the apophyseal joints of human thoracic and lumbar vertebrae. Am J. Phys Anthr 136:318-326. doi:10.1002/ ajpa. 20814

Rodrigues Carvalho C. 2004 Marcadores de estresse ocupacional em populações sambaquieiras do litoral fluminense. Tesis Doctoral inédita. Escola Nacional de Saúde Pública. Fundación Osvaldo Cruz.

Rogers J, Waldron T, Dieppe P, Watt I. 1987. Arthropaties in paleopathology: the basis of classification according to most probable cause. J Archaeol Sci 14:179-193. doi:10.1016/0305-4403(87)90005-7

Rogers J, Shepstone L, Dieppe P. 2004. Is osteoarthritis a systemic disorder of bone? Arthritis \& Rheumatism 50(2):452-457. doi:10.1002/art.20136

Rogers J, Waldron T. 1995. A field guide to joint diseases in archaeology. Baffin Lane: John Willey \& sons.

Rojas-Sepúlveda C, Ardagna Y, Dutour O. 2008. Paleoepidemiology of vertebral degenerative disease in a PreColumbian Muisca series from Colombia. Am J Phys Anthropol 135(4):416-430. doi:10.1002/ajpa.20762

Rothschild B. 1997. Porosity: a curiosity without diagnostic significance. Am J Phys Anthropol 104:529-533. doi:10.1002/(SICI)10968644(199712)104:4<529::AID-AJPA7>3.0.CO;2-M

Säger P. 1969. Spondylosis cervicalis. A pathological and osteoarchaeological study of osteochondrosis in vertebralis cervicalis, arthrosis uncovertebralis and spondylarthrosis cervicalis. Copenhagen: Munksgaard.

Scabuzzo C. 2012. Estudios bioarqueológicos de marcadores de estrés ocupacional en cazadores recolectores pampeanos del Holoceno temprano-medio. Análisis de la serie esqueletal de Arroyo Seco 2. Rev Arg Antropol Biol 14(1):17-31.

Seibel M, Robins S, Bilezikian J. 2006. Dynamics of bone and cartilage metabolism. Nueva York: Elsevier.

Sofaer Derevenski JR. 2000. Sex differences in activityrelated osseous change in the spine and the gendered division of labor at Ensay and Wharram Percy, UK. Am J Phys Anthropol 111:333-354. doi:10.1002/(SICI)10968644(200003)111:3<333::AID-AJPA4>3.0.CO;2-K

Solomon L. 2001. Clinical features of osteoarthritis. En: Ruddy S, Harris E, Sledge C, editores. Kelley's textbook of rheumatology. Philadelphia: Saunders. p. 1409-1418.

Steckel R, Larsen C, Sciulli P, Walker P. 2005. Data collection codebook. The global history of health project. http://www.global.sbs.ohio-state.edu/

Villotte S, Assis S, Alves Cardoso F, Henderson C, Mariotti V, Milella M, Pany-Kucera D, Speith N, Wilczak C, Jurmain R. 2016. Technical note. In search of consensus: terminology for entheseal changes (EC). Int J Paleopathol 13:49-55. doi:10.1016/j.ijpp.2016.01.003

Villotte S, Knüsel C. 2013. Understanding entheseal changes: definition and life course changes. Int J Osteoarchaeol 23(2):135-146. doi:10.1002/oa.2289

Waldron T. 2009. Paleopathology. Cambridge: Cambridge University Press.

Waldron T. 2012. Joint disease. En: Grauer A, editor. A companion to paleopathology. Oxford: Blackwell Publishing. p. 513-530.

Waldron T, Rogers J. 1991. Inter-observer variation in coding osteoarthritis in human skeletal remains. Int J Osteoarchaeol 1:49-56. doi:10.1002/oa.1390010107

Weiss E, Jurmain R. 2007. Osteoarthritis revisited: a contemporary review of aetiology. Int $\mathrm{J}$ Osteoarchaeol 17:437-450. doi:10.1002/oa.889 